\title{
Utilités socioéconomiques et culturelles du Balanites aegyptiaca (L.) Del. (Famille Zygophyllaceae) chez les populations locales de la Région du Ouaddaï au Tchad
}

\author{
ABDOULAYE Brahim ${ }^{1}$, Ali Brahim BECHIR ${ }^{2 *}$, Pierre Marie MAPONGMETSEM ${ }^{3}$ \\ 'Université de N'gaoundéré, Faculté des Sciences, B.P. 454 N'Gaoundéré/Cameroun, Tel. : (+235) 92177160 / 66 \\ 376681 \\ 2 Université des Sciences et de Technologie d'Ati, BP 9957, Ati, Tchad. Tél : (+235) 662542 61/91 0035 74, \\ abrabechir@yahoo.fr \\ 3Université de N'gaoundéré, Faculté des Sciences, B.P. 454 N'Gaoundéré/Cameroun, Tel. : (+237) 777979 96, \\ piermapongahoo.fr \\ *Auteur correspondant Tel. : (+235) 662542 61/ (+235) 910035 74, abrabechir@yahoo.fr
}

Original submitted in on $12^{\text {th }}$ January 2016. Published online at www.m.elewa.orgon $31^{\text {st }}$ March 2017 http://dx.doi.org/104314/jab.v111i1.2

\section{RÉSUMÉ}

Objectif: Cette étude réalisée dans la Région du Ouaddaï au Tchad a pour objectif de comprendre les pratiques et les perceptions paysannes dans l'utilisation de Balanites aegyptiaca.

Méthodes et résultats : des enquêtes socioéconomiques et ethnobotaniques ont été menées auprès d'un échantillon de 172 personnes appartenant à trois villages de la région. Les utilisations de l'arbre par ces populations sont alimentaires, médicinales, fourragères et socioculturelles. Les parties de l'arbre les plus utilisées sont les fruits (100\%), les feuilles $(98 \%)$ et le bois $(66 \%)$. Les jeunes feuilles sont consommées comme légume par 100\% des Maba et Tama, $95,22 \%$ des Massalit et $68,54 \%$ des Mimi. Sur le plan médicinal, B. aegyptiaca est utilisé pour soigner plusieurs affections dont la diarrhée $(59,75 \%)$, la dysenterie $(51,50 \%)$, les maux de poitrine $(40 \%)$, la constipation $(20,38 \%)$ et la Coqueluche $(18,56 \%)$. Les principaux modes de préparation des recettes sont, la décoction $(35,24 \%)$, la trituration $(31,23 \%)$, l'infusion $(24,12 \%)$ et les macérations aqueuses $(10,67 \%)$. Les graines et les épines sont aussi d'une grande importance socio-culturelle. Les activités socio-économiques observées autour des produits de l'arbre concernent la commercialisation des fruits, des graines et du bois qui constitue un intérêt socio-économique certain pour les populations, notamment les femmes.

Conclusion et applicabilité des résultats : Cette étude a montré que les populations de la région du Ouaddaï disposent d'un ensemble de connaissance et de savoir-faire sur le B. aegyptiaca. Les savoirs locaux endogènes identifiés ainsi que les perceptions constituent une base pour le développement de stratégies de conservation et d'utilisation durable de ces ressources génétiques. En conséquence, la disponibilité des produits issus de cet arbre devrait être accrue à travers sa domestication et son introduction dans les systèmes locaux de production paysans existants.

Mots clés : Savoirs endogènes, Balanites aegyptiaca, domestication, Ouaddaï, Tchad. 


\section{Socioeconomic and cultural utilities of Balanites aegyptiaca (L.) Del. for local populations of the Ouaddaï region in Chad ABSTRACT}

Objective: This study done in the region of Ouaddai in Chad aims to assess the farmer practices and perceptions in the use and conservation of Balanites aegyptiaca.

Methodology and results: Socio-economic and ethno botanical interviews were undertaken with a sample of 172 people from three villages of this region. The usages of this tree by local populations are food, medicinal, forage and socio-cultural. The fruits (100\%), leaves (98\%) and wood $(66 \%)$ are the most highly used parts of the tree. Young leaves are consumed as vegetables by $100 \%$ of Maba and Tama, $95.22 \%$ of Massalit and $68.54 \%$ of Mimi. In medicinal terms, B. aegyptiaca is used to treat several affections including diarrhea $(59.75 \%)$, dysentery $(51.50 \%)$, chest pain $(40 \%)$, constipation $(20.38 \%)$ and pertussis $(18.56 \%)$. The main types of recipes preparation were described are decoction (35.24\%), powders (31.23\%), infusion (24.12\%) and aqueous macerations (10.67\%). Seeds and thorns are also a great socio-cultural importance. The socioeconomic activities observed around the tree products concern fruits, seeds and wood marketing which constitute a socio-economic interest for local community and in particular for women.

Conclusion and Application of findings: This study showed that local people in the region of Ouaddai have a wealth of knowledge and expertise on $B$. aegyptiaca. The endogenous local knowledge identified and the perceptions constitute a basis for the development of conservation strategies and sustainable use of these genetic resources. Consequently, the availability of products derived from this three should be increased through his domestication and introduction into the existing local farmer production systems.

Keywords: Endogenous knowledge, Balanites aegyptiaca, domestication, Ouaddaï, Chad

\section{INTRODUCTION}

Les populations rurales sahéliennes ont des connaissances ethnobotaniques traditionnellement très riches grâce aux diversités culturelles et écologiques de l'environnement dans lequel ils vivent (Van den Eyden et al., 1994). Ces connaissances ne sont pas statiques mais dynamiques, et peuvent aider à la domestication des meilleurs individus (Kakudidi, 2004). De nos jours, les ligneux sont très sollicités par les populations sahéliennes en général et celle de la Région du Ouaddaï en particulier. Ces populations trouvent dans la strate ligneuse des fruits et aliments d'appoints, des médicaments, une source d'énergie, des matériaux indispensables à la fabrication d'objets d'utilisation courant et d'augmentation substantielle des revenus des ménages défavorisés (Belem et al., 2008 ; Mapongmetsem et al., 2012). Cette situation entraîne une surexploitation des arbres à usages multiples avec comme conséquence la régression de nombreuses espèces ligneuses (Noubissié-Tiagam et al., 2011). Dans la région du Ouaddaï, les principaux fruitiers " sauvages » très appréciés par les populations locales dont les produits sont rencontrés sur les marchés locaux sont Ziziphus mauritiana Lam., Balanites aegyptiaca (L.) Del., Sclerocarya birrea A. Rich., Diospyros mespiliformis Hochst. ex A. DC., Boscia senegalensis (Pers.) Lam. Ex., Tamarindus indica L., Salvadora persica L., Acacia senegal (L.) Willd et Grewia tenax Forsk. L'implication de ces espèces locales dans l'alimentation, les soins traditionnels et la création des revenus aux populations n'est plus à démontrer (Chevalier et al., 2004 ; Malaise, 2010 ; Mapongmetsem et al., 2010). C'est pourquoi, surexploitées, elles sont aujourd'hui menacées d'extinction et leur faible régénération doublée des pratiques de récolte non durables de leurs produits accélèrent le processus. Concernant Balanites aegyptiaca, objet de la présente étude, bien que l'espèce soit écologiquement considérée comme une espèce résiliente adaptée aux différents types de sols et aux conditions climatiques variées (Hiernaux et al., 2006), les risques de diminution du potentiel de production de l'arbre sont réels. En effet, toutes les parties de la plante (racines, feuilles, fruits, écorces, épines, bois) sont utilisées à des fins alimentaires, thérapeutiques, économiques ou socioculturelles (Walter, 2001 ; Elseed et al., 2002 ; 
Elfeel et Warrag, 2011 ; Chahad et al., 2015). Très sollicitée, $B$. aegyptiaca se trouve ainsi soumis à une pression anthropique permanente à laquelle viennent s'ajouter les défis environnementaux qui menacent dangereusement sa survie. Surexploitée, menacée par les feux, le surpâturage et la sécheresse, cette essence est en nette régression dans la région. Malgré l'importance de cette espèce dans la vie des populations et son utilisation quotidienne, on dispose de très peu d'informations au Tchad sur sa valeur écologique et surtout socioéconomique. Sa chaine de valeur est encore informelle et par conséquent, sa contribution au niveau de vie des populations et de l'économie nationale reste peu ou non connue. Dès lors il est important d'agir pour freiner cette dégradation ou au

\section{MATERIEL ET METHODES}

Description de la zone d'étude : L'étude a été effectuée dans la région du Ouaddaï dont le chef-lieu Abéché constitue un point de convergence du Tchad oriental. La superficie de la zone d'étude est de 29980 $\mathrm{km}^{2}$ pour une population 731679 habitants (INSEED, 2009). Cette zone est située entre le $12^{\mathrm{ème}}$ et le $16^{\mathrm{ème}}$ parallèle Nord, sous l'influence d'un climat de type sahélien (isohyètes 200 et $600 \mathrm{~mm}$ ) avec une longue saison sèche (novembre à mai) et une courte saison des pluies (de juin à octobre). La pluviométrie moyenne annuelle des 10 dernières années est de $545,21 \mathrm{~mm}$ à la station météorologique d'Adré (DGMN, 2017). La température de la région varie selon la saison. En saison sèche froide (décembre à février), les températures journalières se situent en moyenne entre 16 et $35^{\circ} \mathrm{C}$; en saison sèche chaude (mars à avril), les températures oscillent entre 25 et $41^{\circ} \mathrm{C}$; en saison des pluies (juin à août), elles diminuent et varient entre 21 et $31^{\circ} \mathrm{C}$. Le relief, très accidenté est marqué par des affleurements rocheux ainsi que la présence de pierres et des blocs. Les sols sont de faible fertilité à cause des recouvrements sableux et offrent ainsi un potentiel agricole limité. La Région est parcourue par des cours d'eau qui sont en majorité de type non permanent («ouadis») et endoréique, dont la durée des écoulements dépend de la variabilité de la pluviométrie et de leur position topographique. Les caractéristiques climatiques (climat moins stabiliser la situation au profit des générations futures. L'acquisition d'un certain nombre d'informations sur cette espèce forestière locale s'avère donc nécessaires pour sa valorisation et sa conservation. Ces informations indispensables à la domestication de l'espèce sont détenues par les populations locales. C'est pourquoi, pour valoriser le savoir paysan dans la politique de conservation et de gestion durable des ressources naturelles, nous entreprenons cette étude pour connaitre les pratiques et les perceptions des populations de la région du Ouaddaï dans l'utilisation et la conservation de Balanites aegyptiaca. La finalité est de contribuer au développement des stratégies de conservation et d'utilisation durable de ce patrimoine naturel.

sahélien) combinées à celles physiographiques font de la Région du Ouaddaï, une des zones les plus hostiles du pays à cause de la difficulté d'accès à l'eau, du couvert végétal peu fourni, synonyme de ressources limitées, et d'une pluviométrie irrégulière à l'origine des rendements agricoles aléatoires. La végétation, qui est l'expression de ces différents facteurs, se présente sous la forme de steppes herbeuses, arbustives basses à épineux au Nord, à de steppes arborées et des forêts claires ou peu denses à moyennement denses au Sud. Les " ouadis " du Nord-Ouest sont caractérisés par une végétation arbustive souvent claire composée d'espèces comme Acacia tortilis, Acacia mellifera, Acacia laeta, Balanites aegyptiaca, Boscia senegalensis, Dichrostachys cinerea, Ziziphus mauritiana, Sclerocarya birrea, et Salvadora persica. Au Sud sur sols argileux, la végétation devient plus boisée. Tamarindus indica, Anogeissus leiocarpus et Combretum glutinosum forment des forêts galeries assez denses. Les sols sableux à sablo-argileux de fonds des vallées sont en revanche occupés par Acacia seyal, Bauninia rufescens et Acacia nilotica. La strate herbacée sous forme de tapis plus ou moins continu est dominée par Schoenefeldia gracilis, Zornia glochidiata, Cassia obtusifolia et Dactyloctenium aegyptiaca. Les investigations ont concerné trois (3) villages: Arkou, Kokorguin et Mandafana (figure 1). 


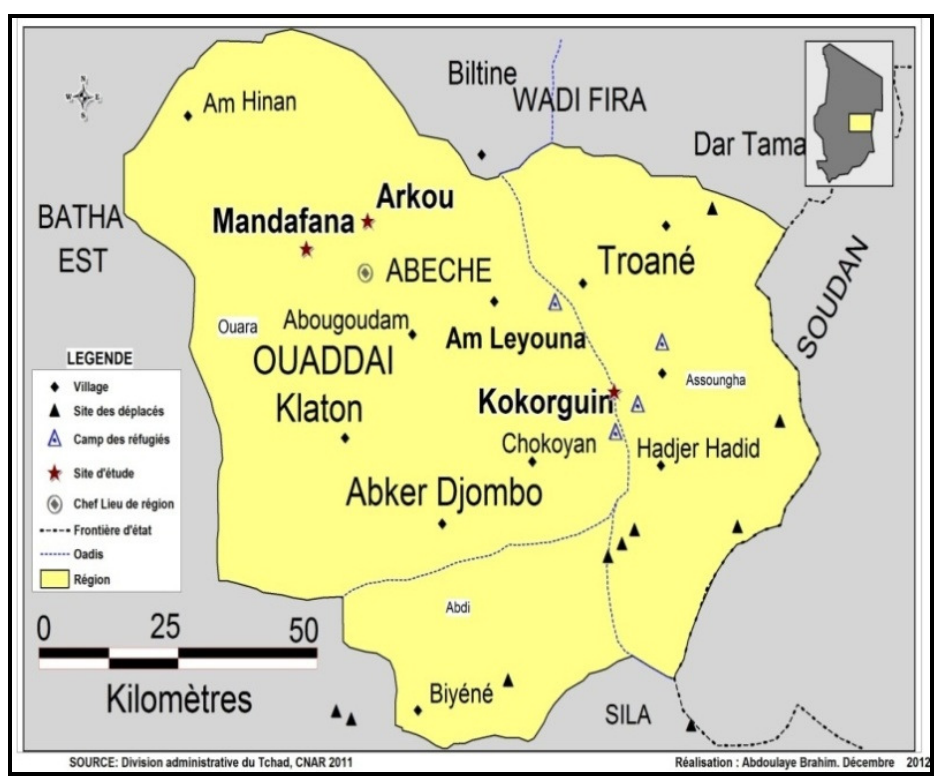

Figure 1 : Carte de localisation du site dans la Région du Ouaddaï au Tchad

Description de B. aegyptiaca : Appelé encore dattier du désert, le Balanites aegyptiaca (L.) Del. est une espèce des zones sahéliennes et soudano-sahéliennes d'Afrique, d'Arabie et de l'Inde. II se rencontre sur une large zone d'Afrique du littoral de la Mauritanie et du Sénégal à la Somalie, et du sud de l'Egypte à la Zambie et au Zimbabwe mais aussi dans les régions chaudes du Congo Démocratique et de l'Angola. C'est un arbuste ou un arbre épineux atteignant 6 à $9 \mathrm{~m}$ de haut. Généralement droit et souvent cannelé, le tronc mesure 30 à $35 \mathrm{~cm}$ de diamètre. Les branches sont étalées de manière irrégulière et à extrémité légèrement retombante formant parfois une cyme arrondie. L'écorce dure, de couleur gris foncé devient rugueuse et profondément striée avec une couronne arrondie ou ovale (Gnoula et al., 2008). Les rameaux et les épines sont vert grisâtre devenant brun clair finement pubérulents à tomenteux, mais généralement glabrescents. Axillaires, droites ou légèrement incurvées, cylindriques avec un bout pointu ou subulé, les épines mesurent 2 à $8 \mathrm{~cm}$ de long et 2 à 6 $\mathrm{mm}$ de diamètre à la base. Les feuilles composées sont subsessiles ou portées par un pétiole d'environ $2 \mathrm{~cm}$ de long. Le dattier du désert est très résistant à la sécheresse et pousse sur tous les types de sol (Arbonnier, 2000).

Collecte de données socio-économiques: Des interviews semi-structurées ont été réalisées auprès de 172 personnes volontaires (94 hommes et 78 femmes) âgés de 35 à 75 ans appartenant aux groupes ethniques Maba, Massalit, Mimi et Tama, des villages Arkou, Kokorguin et Mandafana, entre mai et novembre 2012 (Tableau 1).

Tableau 1 : Répartition des personnes enquêtées selon l'ethnie et le sexe dans la Région du Ouaddaï au Tchad

\begin{tabular}{l|c|c|c|c|c}
\hline & \multicolumn{4}{|c|}{ Ethnies } & Mimi \\
\hline & Maba & Massalit & Tama & Total \\
\hline Ménages & 20 & 17 & 17 & 18 & $\mathbf{7 2}$ \\
\hline Hommes & 27 & 22 & 21 & 24 & 94 \\
\hline Femmes & 21 & 18 & 20 & 19 & $\mathbf{7 8}$ \\
\hline Total & $\mathbf{4 8}$ & $\mathbf{4 0}$ & $\mathbf{4 1}$ & $\mathbf{4 3}$ & $\mathbf{1 7 2}$ \\
\hline
\end{tabular}

Les principaux points du questionnaire ont porté sur l'appellation, les différents usages de cet arbre (alimentation, médecine traditionnelle, commerce et autres usages), les savoirs endogènes, l'état des peuplements et les menaces qui pèsent sur l'espèce. Les interviews individuelles ont été complétées par des entretiens de groupe (5 à 15 personnes). Afin de faciliter la compréhension par les enquêtés, nous nous sommes faits aidés par un guide local qui maîtrise les langues maternelles.

Analyse statistique des données : les moyennes et les déviations standards ont été calculées avec le logiciel 
Excel 2010. L'analyse statistique des données a été réalisée à l'aide du logiciel XLSTAT. Les moyennes

\section{RESULTATS ET DISCUSSION}

Nomenclatures locales de B. aegyptiaca : Les produits forestiers non ligneux (PFNL) les plus valorisés par les populations de la région du Ouaddaï sont ceux issus des espèces fruitières comme Ziziphus mauritiana Lam., $B$. aegyptiaca (L.) Del., Sclerocarya birrea A. Rich., Diospyros mespiliformis Hochst. ex A. DC., Boscia senegalensis (Pers.) Lam. Ex., Tamarindus indica L. et Grewia tenax Forsk. Parmi toutes ces espèces, c'est $B$. aegyptiaca qui a un usage multiple car toutes les parties de la plante sont utilisées. Les populations du Ouaddaï significativement différentes ont été comparées par la méthode de Newman et Keuls au seuil de $\mathrm{P}<5 \%$.

disposent de bonnes connaissances endogènes sur l'espèce qu'elles intègrent dans leur vie sociale, économique et culturelle. Le vocabulaire utilisé pour nommer les espèces ligneuses ou herbacées, leurs parties constituantes, leurs degrés de maturation ou d'exploitation, leurs usages ou leurs produits renseigne sur les connaissances écologiques des ces populations. La multiplicité des noms vernaculaires des plantes et leur existence dans le lexique des noms locaux au sein de chaque groupe ethnique l'attestent si bien (Tableau 2).

Tableau 2 : Liste des principales espèces fruitières de la région du Ouaddaï et leur appellation en langues locales

\begin{tabular}{l|c|c|c|c}
\hline & Maba & Massalit & Mimi & Tama \\
\hline Balanites aegyptiaca & Mallak & Guinda & Kamâra & Hounoud \\
Ziziphus mauritiana & Kouchak & Kourtcha & Nouma & Kaguit \\
Sclerocarya birrea & Goundok & Goundo & Douro & Karat \\
Diospyros mespiliformis & Lilii & Alili & - & Halit \\
Boscia senegalensis & Ayinga & Berté & Dabang & Tabit \\
Tamarindus indica & Koundjii & Koundji & Dongour & Bot \\
Grewia tenax & Kabayinga & Tabayinga & Amderang & Bakkoulé \\
\hline
\end{tabular}

Utilisation de $B$. agegyptiaca par les 4 groupes ethniques : Les utilisations de l'arbre par les populations locales sont multiples: alimentaires, médicinales, fourragères et socioculturelles. Cependant, le taux d'utilisation varie selon les organes. Ainsi, $100 \%$ des personnes enquêtées affirment utiliser le fruit de $B$. aegyptiaca contre $98,23 \%$ pour les feuilles, $78,87 \%$ pour le bois, $11,32 \%$ pour les racines, $10,23 \%$ pour les écorces et seulement 1, 15\% pour les épines (Figure 2).

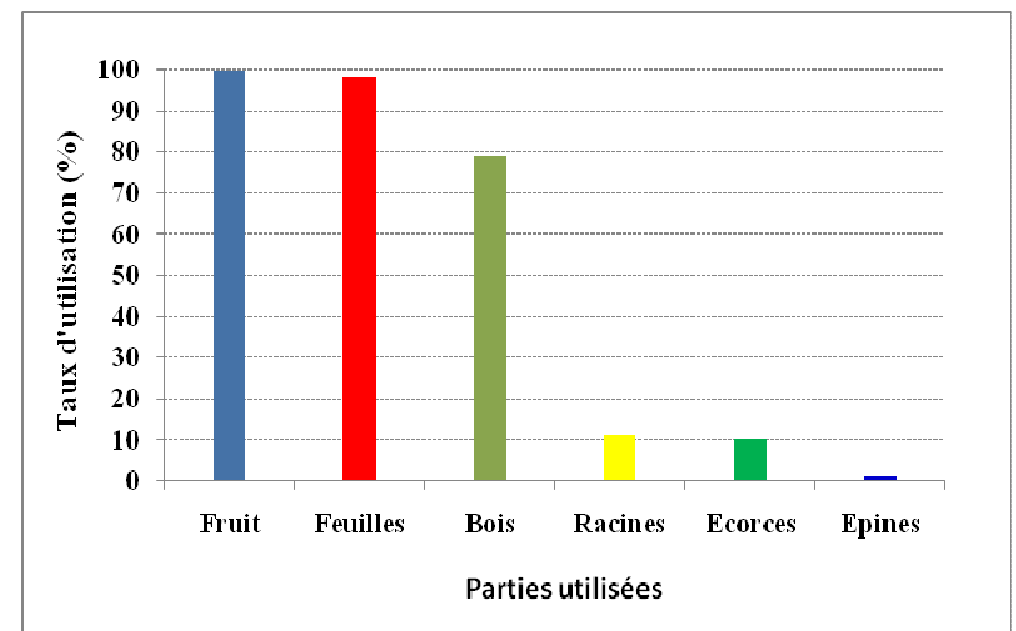

Figure 2 : fréquences d'utilisation des différentes parties de $B$. aegyptiaca dans la Région du Ouaddaï

Les enquêtes ethnobotaniques menées auprès des populations locales ont aussi montré la reconnaissance et l'intérêt des valeurs culturelles, économiques et sociales de l'espèce (100\% des personnes enquêtées). Les populations locales disposent donc d'un savoir-faire ainsi que d'importantes connaissances sur la plante. 
Usages alimentaires: Les usages alimentaires de $B$. aegyptiaca concernent d'abord le fruit $(100 \%)$ dont les parties les plus importantes sont la pulpe et le noyau. L'utilisation de la pulpe dans la préparation du jus a été citée par $62,56 \%$ des enquêtés chez les Maba, 78,56\% chez les Massalit, $12,28 \%$ chez les Tama et $6,45 \%$ chez les Mimi. En effet, la pulpe est conservée sous forme de pâte appelée " Serné » en langue locale. Elle entre dans la préparation d'une bouillie très prisée appelée « Bourdoubourdou » par les Massalit et « Angeli » par les Maba. En outre, chez les Massalit (100\%), la pulpe est également utilisée dans la préparation de la sauce. Macérés dans l'eau, la pulpe du fruit remplace aussi le sucre dans la préparation des bouillies chez le même groupe ethnique. En effet, les valeurs nutritionnelles du fruit ainsi que son utilisation dans l'alimentation humaine et animale sont reconnues par plusieurs auteurs (Farid et al., 2002 ; Chapagain et al., 2009; Hanan et al., 2009 ; Elfeel, 2010; Sagna et al., 2014). Les caractéristiques biochimiques du pulpe du fruit de $B$. aegyptiaca montrent une qualité nutritionnelle acceptable avec une valeur énergétique élevée à cause de la présence des sucres (Sagna et al., 2014 ; Soloviev et al., 2004). Les drupes et les amandes du fruit de $B$. aegyptiaca sont aussi consommées par l'ensemble des populations enquêtées $(100 \%)$. Commercialisées dans les différents marchés hebdomadaires de la région, les amandes sont généralement consommées fraîches par succion. Appelée communément «kournaka » en langue locale, l'amande est consommée après cuisson. En effet, après une première ébullition qui dure une demi-heure environ, les amendes sont retirées puis triturées à la main afin d'enlever l'enveloppe blanche. Elles sont encore remises à cuire dans une nouvelle eau pendant plusieurs heures afin d'atténuer leur amertume. Séchées au soleil, elles sont alors consommées bien que certaines gardent une partie de leur goût initial. L'utilisation des amandes de $B$. aegyptiaca dans l'alimentation humaine est aussi rapportée par Belge et al. (2006). De l'huile extraite de l'amande est aussi utilisée dans la préparation de la sauce chez les Massalit et les Tama (32,8\% et $16,32 \%$ respectivement). L'utilisation de l'huile de $B$. aegyptiaca en cuisine a été également signalée par Mohammed et al.
(2002). En effet, très similaire aux huiles de sésame et de l'arachide (Abu Al-Futu, 1983), cette huile est riche en protéines (Elfeel, 2010) et peut être également utilisée comme biocarburant (Chapagain et al., 2009). Les jeunes feuilles de $B$. aegyptiaca sont également consommées comme légume par $100 \%$ des Maba et Tama, $95,22 \%$ des Massalit et $68,54 \%$ des Mimi. Mélangées à la pâte d'arachide ou de sésame ou parfois à un peu de farine de mil, elles sont utilisées pour la préparation de la sauce. Selon les Tama $(97,12 \%)$ et les Maba $(87,54 \%)$, les feuilles bouillies, séchées et réduites en poudre sont également ajoutées à la farine de mil pour la préparation de la bouillie ou du couscous en période de disette. Plusieurs travaux menés en Afrique de l'Ouest ont montré I'utilisation du fruit et des feuilles de $B$. aegyptiaca dans la consommation humaine et animale (Cook et al., 2000 ; Arbonnier., 2002 ; Belge et al., 2006). Seul, l'endocarpe de consistance ligneuse n'a aucune utilité alimentaire directe dans la région. En outre, $B$. aegyptiaca est également un bon fourrage pour les animaux qui apprécient particulièrement les feuilles, les jeunes pousses et les fruits. Les parties les plus appétées sont par ordre d'importance: les feuilles $(100 \%)$, les fruits $(96,4 \%)$, les jeunes rameux $(58,23 \%)$ et les jeunes pousses $(56,34 \%)$. Les fruits sont exclusivement consommés par les petits ruminants lorsqu'ils tombent au sol. La consommation de ces différents organes de l'arbre par les animaux est également rapportée par Chevalier et al. (2004) et Kaboré-Zoungrana et al. (2008). Selon les mêmes auteurs, l'apport de l'arbre dans l'affouragement du bétail est particulièrement élevée surtout durant la saison sèche.

Usages médicinaux : dans la région du Ouaddaï, toutes les parties de l'arbre sont utilisées en pharmacopée traditionnelle pour soigner plusieurs affections humaines. Les résultats de nos travaux ont montré que sept (7) maladies sont connues pour être soignées par le $B$. aegyptiaca dont trois sont très fréquentes: la diarrhée $(59,75 \%)$, la dysenterie $(51,50 \%)$, les maux de poitrine $(40 \%)$, la constipation $(20,38 \%)$, la Coqueluche $(18,56 \%)$, les plaies $(12,25 \%)$ et les morsures de serpent $(5,24 \%)$ (Figure 3). 


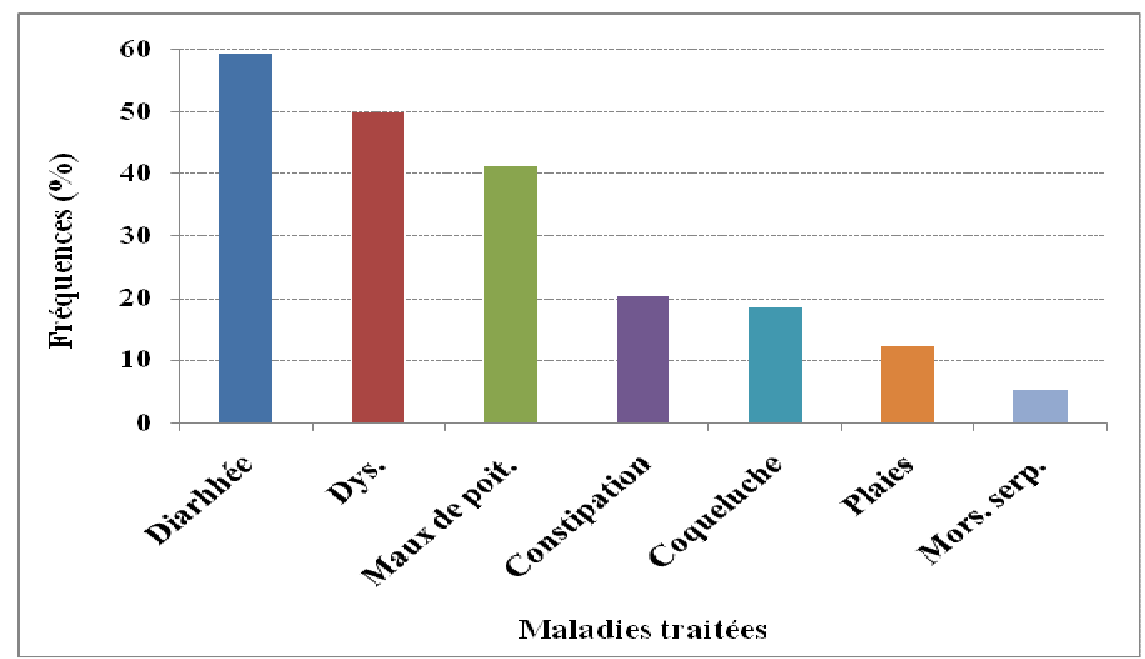

Figure 3 : Fréquences d'utilisations médicinale et pharmacologique de $B$. aegyptiaca (L.) Del. dans les soin des divers affections humaines dans la région du Ouaddaï au Tchad Dys. $=$ Dysenterie $;$ Maux de poit. $=$ Maux de poitrine $;$ Mors. Serp. $=$ Morsure de serpent

L'utilisation de la plante dans le traitement d'autres affections (constipation, les hémorroïdes, les brulures, les plaies) a également été rapportée. Concernant le fruit, toutes les parties à savoir l'épicarpe $(68,21 \%)$, la pulpe $(63,12 \%)$ et les amandes $(38,21 \%)$ sont utilisées pour les soins. Nos résultats corroborent ceux obtenus par Saboo et al. (2014). Les Mimi (78,65\%), les Maba (76,32\%) et les Tama (32,43\%) utilisent la pulpe du fruit pour soigner les maux de ventre et la décoction de l'épicarpe pour soigner les maux de reins. Le recours à la pulpe du fruit pour soigner les maux de ventre est également rapporté par Hamilton et El Naïm (2000) et Gning et al. (2014). La majorité des groupes ethniques enquêtés $(98,02 \%$ des Maba, $97,65 \%$ des Tama et $98,43 \%$ des Massalit) utilisent l'huile extraite de l'amande pour soigner la coqueluche et les maux de poitrine. Les propriétés anti cancérigène de l'huile de $B$. aegyptiaca ont été également rapportées par Hanan et al. (2009). Les poudres de l'écorce et des racines sont aussi utilisées comme laxatives et pour soigner les maux de ventre et la constipation chez les Maba (21,43\%), les Massalit $(19,12 \%)$, les Tama $(11,43 \%)$ et dans une moindre mesure chez les Mimi $(9,87 \%)$. Les racines sont utilisées comme laxatives (36,13 et 28,23\% des Maba et Massalit respectivement), contre les maux de ventre par les Maba
$(22,12)$, les Massalit $(20,01 \%)$, les Tama $(12,03 \%)$ et les Mimi $(7,76 \%)$. Les racines sont aussi utilisées par les Massalit $(52,23 \%)$, les Tama $(43,10 \%)$, les Maba $(23,12 \%)$ et les Mimi $(3,76 \%)$ pour soigner la constipation, les infections et les morsures de serpent. Cependant, bien qu'utilisées pour soigner des affections, les racines demeurent avec les épines et les feuilles, les organes les moins utilisées en pharmacopée traditionnels dans la région. La faible utilisation des racines dans les soins traditionnelles par les populations du Ouaddaï corrobore les résultats obtenus par Gning et al. (2014). Cependant, ils sont contraires à ceux de Cissé et al (2016) qui signalent en revanche une forte utilisation des racines de B. aegyptiaca par les bergers peuls du Ferlo au Sénégal pour soigner plusieurs pathologies. Plusieurs types de préparations ont été également décrites par les différents groupes ethniques et les parties de la plante sont utilisées fraîches, séchées ou réduites en poudres. Les principaux modes de préparation des recettes sont, la décoction $(35,24 \%)$, la trituration $(31,23 \%)$, l'infusion $(24,12 \%)$ et les macérations aqueuses $(10,67 \%)$ (Figure 4). La boisson est la principale voie d'administration $(85,21 \%$ des enquêtés) contre $24,79 \%$ pour les applications externes (bains, pansements, scarifications, gargarismes, fumigations, massages, purges). 


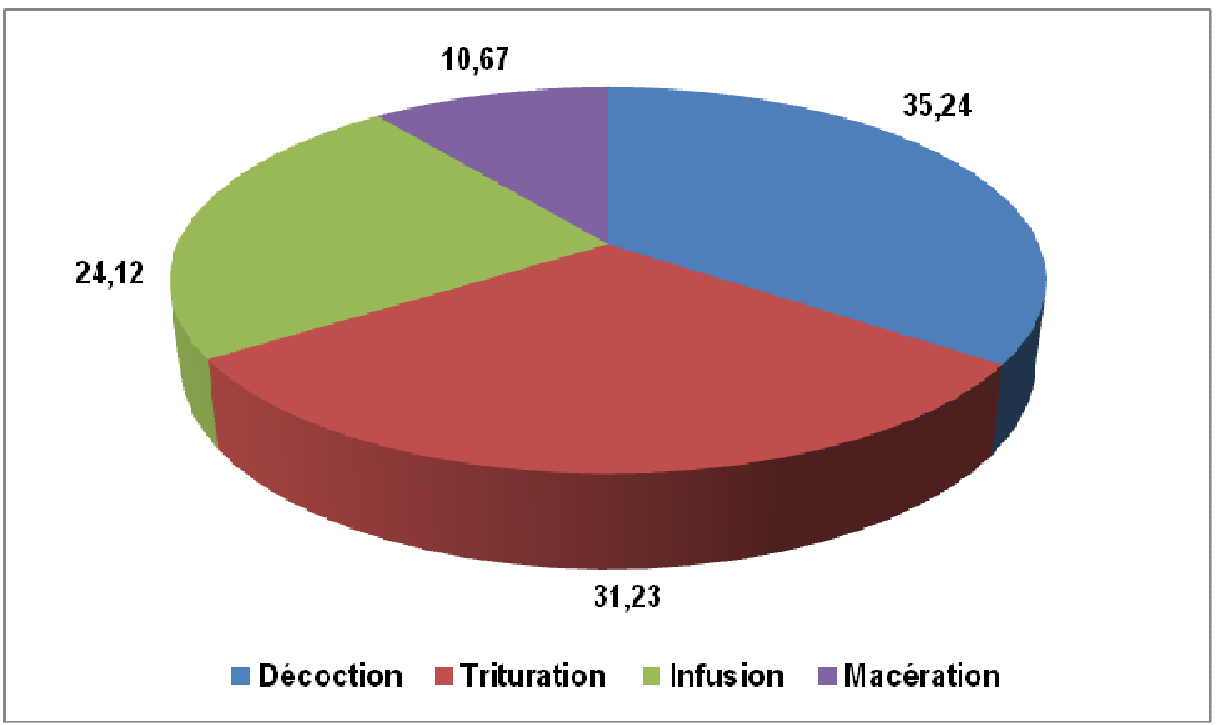

Figure 4 : Fréquences des différents modes de préparation des remèdes (\%)

Commercialisation des produits de B. aegyptiaca:

Les activités socio-économiques observées autour des produits de l'arbre concernent la commercialisation des fruits et du bois $(69,89$ et $68,72 \%$ respectivement) suivi par celle des graines (57\%). Le commerce des feuilles de $B$. aegyptiaca par les différents groupes ethniques est faiblement pratiqué $(8,71 \%)$. Toute fois, les graines sont beaucoup plus commercialisées par les Mimi (60,94\%) alors que les premiers vendeurs du bois de $B$. aegyptiaca sont les Mimi (80,48\%) et les Massalit (77,50\%) (Tableau 3).

Tableau 3 : Proportion des vendeurs (\%) des produits de B. aegyptiaca dans la Région du Ouaddaï au Tchad

\begin{tabular}{l|c|c|c|c|r}
\hline \multicolumn{1}{c|}{ Parties vendues } & Maba & Massalit & Mimi & Tama & \multicolumn{1}{c}{ Moyenne } \\
\hline Feuilles & $10,41 \mathrm{~b}$ & $6,30 \mathrm{c}$ & $12,19 \mathrm{~b}$ & $5,97 \mathrm{c}$ & $8,71 \pm 2,66 \mathrm{c}$ \\
Fruits & $60,46 \mathrm{a}$ & $72,50 \mathrm{a}$ & $73,17 \mathrm{a}$ & $68,75 \mathrm{a}$ & $68,72 \pm 5,05 \mathrm{a}$ \\
Graines & $56,25 \mathrm{a}$ & $55 \mathrm{~b}$ & $60,97 \mathrm{a}$ & $55,81 \mathrm{~b}$ & $57,00 \pm 2,33 \mathrm{~b}$ \\
Bois & $54,16 \mathrm{a}$ & $77,50 \mathrm{a}$ & $80,48 \mathrm{a}$ & $67,44 \mathrm{a}$ & $69,89 \pm 10,28 \mathrm{a}$ \\
\hline
\end{tabular}

$a, b, c$ : dans une même colonne, les moyennes suivies d'une lettre différente sont significativement différentes au seuil de $P<5 \%$

Ces produits sont vendus sur les marchés locaux et le marché régional d'Abéché, chef lieu de la Région. L'apport de leur vente dans les revenus des ménages paysans est considérable. Les fruits sont vendus en tas, en « coros » $(2 \mathrm{~kg})$ ou en sac de $100 \mathrm{~kg}$. Les prix de vente du tas varient entre $25 \mathrm{FCFA}$ en période de récolte et 50 FCFA en période de rupture. Le prix du « coro » du fruit de $B$. aegyptiaca varie entre 200 et 450 FCFA. La galette de la pulpe de $B$. aegyptiaca ou "Serné » est vendue à 100 FCFA l'unité. Les prix des amandes ou "Kournaka » vendues aux marchés locaux varient entre 100 FCFA la petite calebasse ou "kasse » en langue locale, à 1000 FCFA le « Coros » ou grande tasse de $2 \mathrm{~kg}$ (Figure 5). 


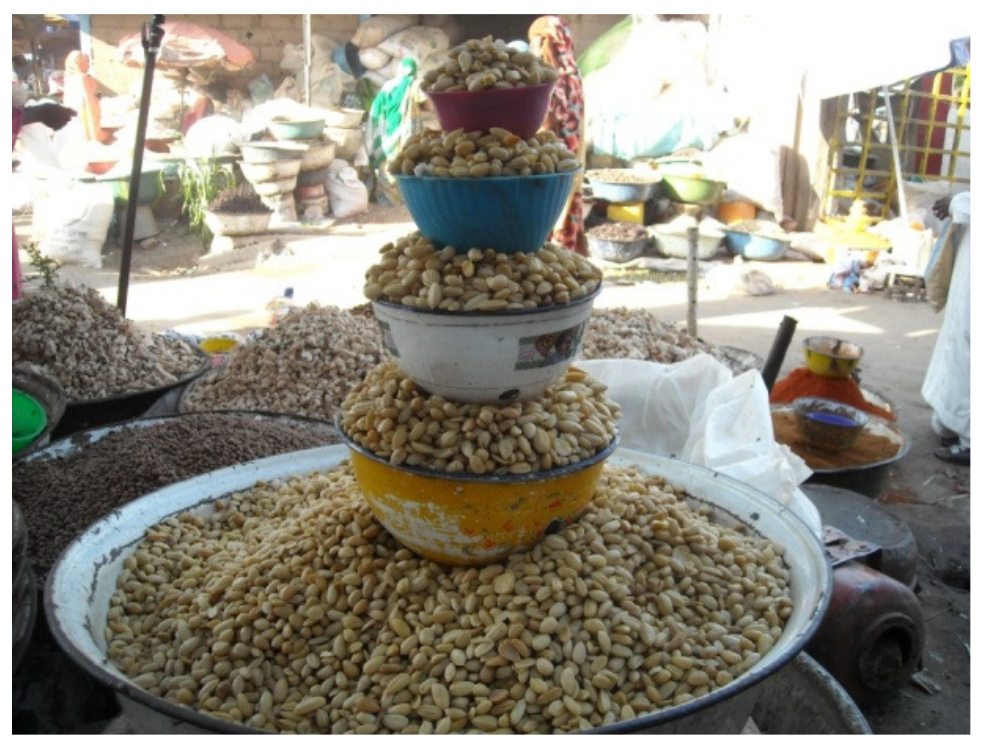

Figure 5 : Amandes de B. aegyptiaca ou « Kournaka » prêtes à la consommation vendues au marché d'Arkou.

La commercialisation des fruits présente un intérêt socioéconomique certain pour ces populations. La commercialisation des produits de cet arbre présente donc un intérêt socio-économique certain pour les populations, notamment pour les femmes. La haute valeur commerciale du fruit et de la graine du $B$. aegyptiaca a été également rapportée par Faye et al. (2010) et Abasse et al. (2010). Très résistant et à multiples usages, une grande consommation du bois de $B$. aegyptiaca a été rapportée par les populations enquêtées (100\%). En effet, il est très utilisé comme matériau de construction des cases et autres abris $(68,12 \%)$, les enclos $(56,23 \%)$ et la clôture $(18,12 \%)$. Le bois est aussi utilisé comme combustible pour la cuisson
$(28,56 \%)$ et le chauffage $(13,32 \%)$. II sert pour la fabrication des mortiers $(98,21 \%)$ et également pour la confection des tablettes coraniques ou « Loh » en langue locale $(95,02 \%)$ (Figure 6a), des selles et des manches à outils $(79,67 \%)$. L'utilisation du bois de $B$. aegyptiaca dans la fabrication du gourdin ou "soforok » en langue locale (Figure $6 b$ ), une sorte de bâton aplati très utilisé pour l'autodéfense et la chasse aux rongeurs et aux pintades en milieux Maba et Massalit a été citée par $86,76 \%$ des personnes enquêtées. Les mêmes utilisations du bois de $B$. aegyptiaca ont été également rapportées par Sagan et al. (2014). L'écorce du tronc de $B$.aegyptiaca est également utilisée comme savon pour laver les habits (12,08\% des personnes enquêtées).

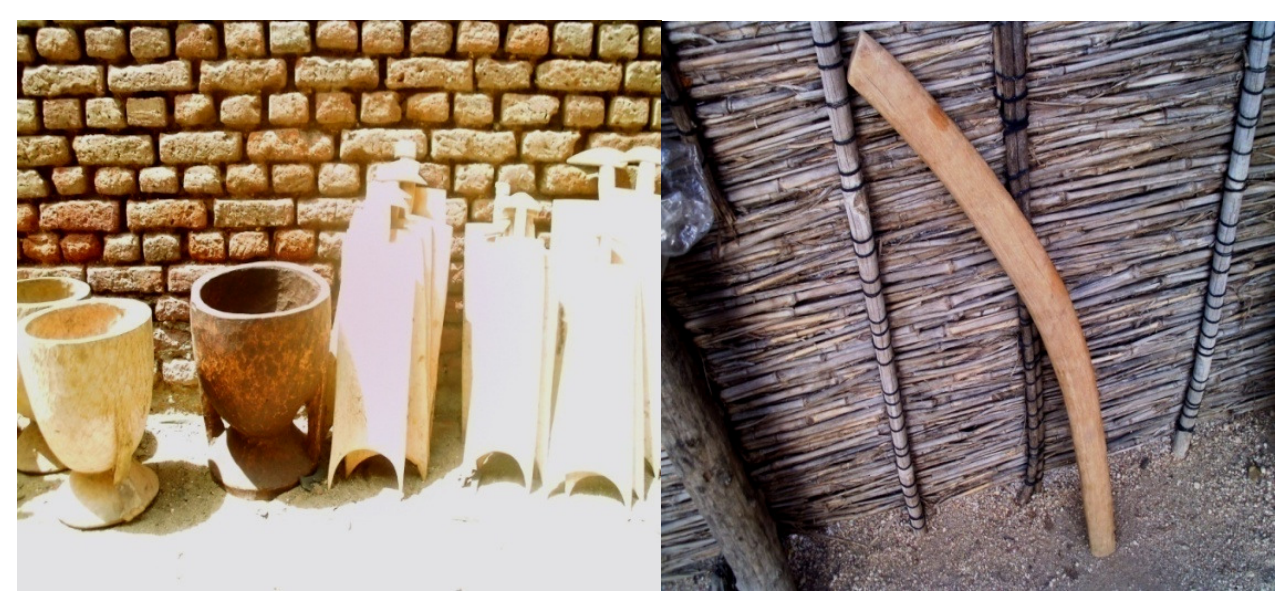

Figure 6 : Mortiers et tablettes coraniques (a) ; « Soforok» ou gourdin (b) faits à base du bois de B. aegyptiaca dans le Ouaddaï au Tchad. 
En raison de ces utilisations multiples, le bois de $B$. aegyptiaca constitue une importante source de revenus pour les populations locales et l'arbre se trouve ainsi soumis à une très forte pression anthropique. Dans les marchés locaux, le prix du bois varie selon la saison. En effet, la charge d'un âne $(60 \mathrm{~kg})$ est vendue à 1500 FCFA en saison sèche et 2500 FCFA en saison de pluie. Le prix de la charge d'un âne en bois de $B$. aegyptiaca élevé en saison des pluies se justifie par le fait que l'accès au lieu de récolte pendant cette saison est très difficile. La variation du prix du bois de $B$. aegyptiaca selon la saison a été également signalée au Mali (Kouyaté et al., 2002). Le prix du bois a également varié selon la distance parcourue pour la collecte $(68,03 \%$ des personnes enquêtées).

Importance socioculturelle des produits de l'arbre : Certaines parties de l'arbre servent à la fabrication des objets de grandes valeurs sociale et culturelle. Les graines de $B$. aegyptiaca sont utilisées par les Maba $(78,65 \%)$ pour confectionner des chapelets pour les prières (Figure 7).

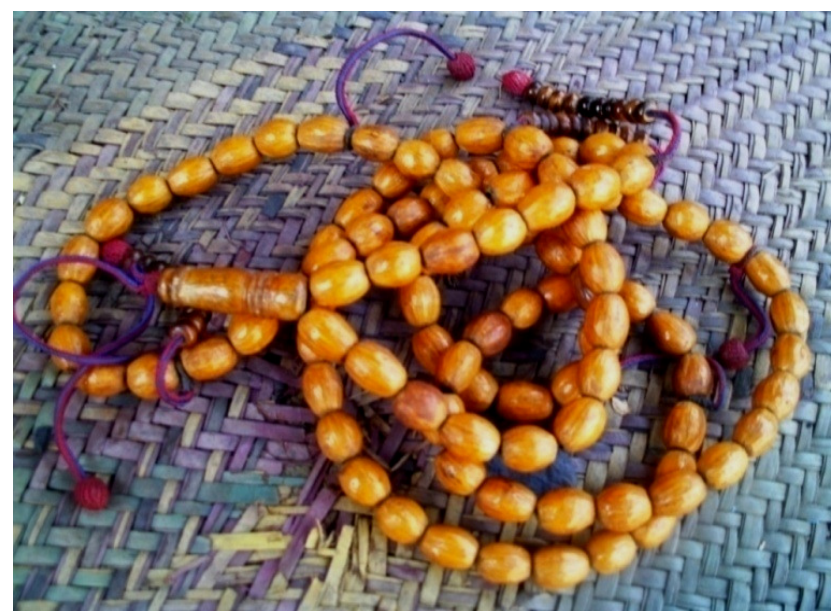

Figure 7 : Chapelet pour la prière à base de graines de $B$. aegyptiaca dans le Ouaddaï au Tchad

Les épines sont également utilisées par le même groupe ethnique $(32,45 \%)$ pour le tatouage des lèvres et de la gencive supérieure des jeunes filles. Ces parties deviennent noires après traitement. Cette pratique, très ancienne a un double intérêt chez les jeunes filles Maba : initiatique car elle marque le passage de la jeune fille à l'âge de la puberté (entre 14 et 15 ans) et esthétique car utilisée pour rendre la jeune fille belle. Les pratiques de tatouages des lèvres et des gencives par les jeunes filles de la région ont été cependant combattues dès le départ par les religieux qui les considèrent comme une déformation de la créature divine qui est la femme. Elles furent ensuite interdites en 1970 par le Front de
Libération Nationale du Tchad (FROLINAT), mouvement politico-militaire. Elles ne sont donc plus pratiquées de nos jours et les séquelles des tatouages sont aujourd'hui perceptibles sur les lèvres et les gencives de certaines femmes âgées de la région. En revanche, cette pratique très ancienne n'est pas observée chez les autres groupes ethniques étudiés (Massalit, Tama et Mimi). En outre, l'utilisation des épines dans la fabrication de la muselière à veau ou « Magnâ » en langue locale (Figure 8 a et b), un instrument utilisé pour sevrer les veaux et les poulains a été rapportée par $93,02 \%$ des Maba, 91,43\% des Massalit, $73,78 \%$ des Mimi et $68,15 \%$ des Tama. 

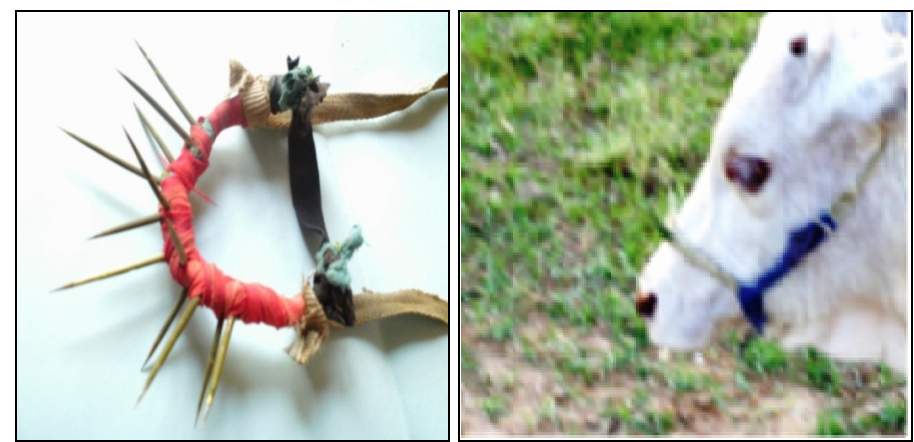

Figure 8 : Muselière à veau ou « Magnâ » fabriquéà base d'épines de B. aegyptiaca (a) et « Magnâ » porté par un veau (b) dans le Ouaddaï au Tchad

\section{CONCLUSION}

Les trois (3) terroirs étudiés renferment une grande diversité floristique parmi laquelle des espèces à usage multiple dont $B$. aegyptiaca qui a une utilisation très ancienne. Les connaissances et le savoir-faire des populations locales sur les plantes en général et sur $B$. aegyptiaca en particulier se lisent à travers le vocabulaire utilisé pour nommer les espèces ligneuses ou herbacées, leurs parties constituantes, leurs degrés de maturation ou d'exploitation, leurs produits ou leurs usages. L'étude a également montré l'utilisation de l'arbre par les populations locales à des fins alimentaires, médicinales, socio-économiques ou culturelles. Le fruit et le bois sont les parties les plus prisées de l'arbre. Les usages alimentaires de $B$. aegyptiaca concernent le fruit dont les parties les plus importantes sont la pulpe et le noyau, et les jeunes feuilles consommées comme légumes ou fourrages. Toutes les parties de l'arbre sont utilisées en pharmacopée traditionnelle pour soigner plusieurs

\section{RÉFÉRENCES BIBLIOGRAPHIQUES}

Abasse T, Weber JC, Katkore B, Boureima M, Larwanou M, Kalinganire A, 2011. Morphological variation in B. aegyptiaca (L.) Del. Fruits and seeds withing and among parkland agroforests in eastern Niger. Agrofor. Syst., 81 : 57-66.

Abu-Al-Futuh IM, 1983. Balanites aegyptiaca: an unutilized raw material potential ready for agroindustrial exploitation. UNIDO Document no 12419 project TF/INT/77/021. UNIDO of the United Nations.

Arbonnier M, 2000. Arbre arbustes et lianes des zones sèches de l'Afrique de l'Ouest. CIRAD (Montpellier) et Muséum National d'Histoire Naturelle (Paris), $574 \mathrm{p}$.

Belem B, Smith OC, Theilade I, Bellefontaine R, Guinko S, Mette Lykke A, Diallo A, Boussim Jl. Identification des arbres hors forêt prefers des affections humaines. Les activités socio-économiques observées autour des produits de $B$. aegyptiaca concernent la commercialisation des fruits, des graines et $\mathrm{du}$ bois. Les graines et les épines sont aussi d'une grande importance socio-culturelle. Ainsi, les multiples avantages qu'apporte $B$. aegyptiaca aux populations de la région du Ouaddaï militent en faveur d'une plus grande promotion des techniques de conservation et de valorisation auprès des populations rurales. Les savoirs paysans recensés, peuvent constituer une source d'inspiration pour le développement de stratégies de conservation et d'utilisation durable des ressources génétiques. En conséquence, l'étude des niveaux de prélèvements par les populations locales, la description de sa filière de commercialisation compléteront cette approche, afin d'aboutir à sa domestication et son introduction dans les systèmes de production paysans existants.

populations du Sanmatenga (Burkina Faso), Bois et For. Trop. 298 (2008) : 53-64.

Belge G, Diallo I, Hveem B, 2006. Les plantes sauvages du Sahel malien KARTHALA Edition, $92 \mathrm{p}$.

Breye C, 2004. Les aménagements hydro agricoles au Tchad: des tentatives de réduction des crises alimentaires (Exemple de paramètre irrigué de Mara), Mémoire de maîtrise en géographie Université de N'djamena (Tchad), $15 \mathrm{p}$.

Chahad AM, Michalet S, Béchir AB, Abdelsalam T, Nkongmeneck BA, Dijoux-Franca MG, 2015. Medicinal Plants from the Ouaddaï Province (Chad): An Ethnobotanical survey of plants used in traditional medicine. The Journal of Alternative and Complementary Medicine, 21 (9) : 569-577.

Chapagain BP, Hariv Y, Zeev W, 2009. Desert date (Balanites aegyptiaca) as an arid lands 
sustainable bioresource for biodiesel. Bioresour. Technol., 100 : 1221-1226.

Cissé A, Gueye M, Ka A, Ndiaye F, Koma S, Akpo LE, 2016. Ethnobotanique des plantes médicinales chez les bergers peuls de Widou Thiengoly de la commune de Téssékéré (Ferlo-Nord Sébégal). Journal of Applied Biosciences, $98: 9301-9308$.

Chevallier MH, Vaillant A, Bensaid S, Sahki-Boutamine $R$, Diallo OB, Sanou J, Bouguedoura N, Babin $D, 2004$. Impact des pratiques humaines sur la conservation et la gestion in situ des ressources forestières : cas d'Acacia tortilis raddiana et de Balanites aegyptiaca. CSFD, Rapport du projet $n^{0} 57,68 \mathrm{P}$.

Direction Générale de la Météorologie Nationale (DGMN), 2017. Données pluviométriques de la ville d'Adré (2003-2013).

Dupriez H, De Leneer P, 1987. Jardin et Verger d'Afrique. Terre et vie. L'Harmattan-Apica Enda. GTA. 354 p.

Elfeel AA, 2010. Variability in Balanites aegyptiacaVar. Aegyptiaca seed kernel oil, protein and minerals contents between and within locations. Agric. J. North Am., (ABJNA), 1 (2): 170-174.

Elfeel AA, Warrag El, 2011. Use and Conservation status of Balanites aegyptiaca (L.) Del. (Hegleig Tree) in Sudan: local People Perspective. Asian Journal of Agicultural Sciences 3 (4):286-290.

Farid $\mathrm{H}$, Haslinger $\mathrm{E}$, Kunert $\mathrm{O}$, Wegner $\mathrm{C}$, Hamburger $M, 2002$. New steroidal glycosides from Balanites aegyptiaca. Helv. Chim. Acta, 88 (4) : 1019-1026.

Faye MD, Weber JC, Mounkoro B, Dakouo J-M, 2010. Contribution of parkland tree and shrub species to village livelihoods: a case study from Mali. Develop Pract 20:428-434.

Gning ON, Sarr O, Akpo LE, 2014. Richesse de la pharmacopée malinké: rôle médicinal de l'arbre aà Khossanto : (Kédougou, Sénégal oriental). Journal of Applied Biosciences 74 :6043-6058

Gnoula C, Mégalizzi V, De nève $N$, Sauvage $S$, Ribaucour $F$, Guissou P, Duez P, Dubois J, Ingrassia L, Le Franc F, Kiss R, Mijatovic T. 2008. Balanitin-6 and-7: Diosgenyl saponins isolated from Balanites aegyptiaca (L.) Del. Display significant anti-tumor activity in vitro and in vivo. Int J. Oncol. $32: 5-15$.

GTZ, 1998. Plan de développement. Le Ouaddaï/Biltine vers l'an 2005. VAM+M tE UNITS, WFP CHAD. $30 \mathrm{p}$.
Guinko S, Pasko LJ, 1992. Haversting and marketing of edible products from local Woody Species in Zitenga, Burkina Faso. UNASYLVAn ${ }^{\circ} 168$, Aride Zone Forestry. 43 (1): 47-56.

Hamilton JG, El Naiem DA, 2000. Sugar in the gut of the Saudfly Phlebotamus orientales fron Dender National Park, Eastern Sudan. Med. Vet. Entomol. 14: 64-70.

Hanan A, Al-Ashaal AF Ayman, Adb El-Aziz MM, Ali MA, 2009. Phytochemical investigation and medicinal evaluation of fixed oil of Balanites aegyptiaca fruits (Balanitaceae). J. Ethnopharmacol. (3): 495-501.

Kaboré-Zoungrana C., Diarra B., Adandadjan C., Savadogo S., 2008. Valeur nutritive de Balanites aegyptiaca (L.) Del. pour l'alimentation des ruminants. Livestock Res. For Rural Dev. 20 (4).

Kakudidi EK, 2004. Folk plant classification by communities arrond Kibale National Park. Western Uganda. Africa Journal of Ecology. 114-118.

Kouyaté A.M., Van Damme P., 2002. Caractères morphologiques de Detarium microcarpum Guill et Perr. AU Sud du Mali, Fruits 57: 231-238.

Kouyaté AM, Guindo F, De Groote DS, Decaluwe E, Diawara H, Diatta L, Van Damme P, 2010. Variabilité morphologique du tamarinier (Tamarindus indica) au Mali. Scripta Bot. Belg., $46: 253 \mathrm{p}$.

Malaise $F, 2010$. How to live and survive in Zambezian open forests. Gembloux, Belgium: les Presses agronomiques de Gembloux.

Mapongmetsem PM, Kapchie V, Tefempa HB, 2012. Diversity of fruit tree species and sustairability of the huehood in the Northern of Cameroon. Ethiopian Journal of Environmental and Management. 5 (1): 37-43.

Mapongmetsem PM, Tsingsabe O, Keumeze V, Damba A, 2010. Utilisation et commercialisation des produits forestiers non ligneux par les communautés locales en zone soudanienne. Com. AETFAT, Antananarivo, Madagascar. 12 p.

Mohammed AM, Wolf W, Spiess WE, 2002. Physical, morphological and chemical characteristics, oil recovery fatty acid composition of Balanites aegyptiaca Del Kernels. Plant Food Human Nutrition. 57 : 179-189.

Noubissié-Tchiagam JB, Ndzié JP, BelleFontaine R, Mapongmetsem PM. Multiplication végétative de Balanites aegyptiaca (L.) Del., Diospyros 
mespiliformis Hochst. ex. A. Rich. et Sclerocarya birrea (A.Rich.) Hochst. au nord du Cameroun. Fruits (2011), Vol. $66: 327-341$

Saadou M, Soumana I, 1993. Plantes alimentaires cultivées et spontanées et recettes culinaires du Niger, Université des Nations Unies, PRMA. 27 p.

Shweta Saboo S, Rani Chavan W, Ganesh Tapadiya G, Khadabadi SS, 2014. An Important Ethnomedicinal Plant Balanites aegyptiaca Del. American Journal of Ethnomedicine, 2014, 1 (3): 122-128.

Sagna MB, Diallo A, Sarr PS, Ndiaye O, Goffner D, Guisse A, 2014. Biochemical composition and nutritional value of Balanites aegyptiaca (L.) Del fruit pulps from Northen Ferlo in Senegal. African Journal of Biotechnology, 13 (2):336342.

Soloviev P, Niang TD, Gaye A, Totte A, 2004. Variabilité des caractères physic-chimiques des fruits de trios espèces ligneuses de cueillette, récoltés au Sénégal: Adansonia digitata, Balanites aegyptiaca et Tamarindus indica. Fruit, 59 : 109119.

Van den Eyden V, Van Damme P, De Wolf P, 1994. Inventaire et modelage de la gestion du couvert végétal pérenne dans une zone forestière $d u$ Sud du Sénégal. Rapport final. Partie C, Ethnobotanique. Université de Gent, Belgique. 33-39p.

Walter S, 2001. Non-woods forest products in Africa: a regional and national overview. Working paper FOPW/01/1. Forestry Department. 32p. 